\title{
Efficiently Matching Proximity Relationships in Spatial Databases
}

\author{
Xuemin $\operatorname{Lin}^{1 \star}$, Xiaomei Zhou ${ }^{1}$, and Chengfei Liu ${ }^{2}$ \\ 1 School of Computer Science and Engineering \\ University of New South Wales, Sydney, NSW 2052, Australia \\ $\{$ lxue, xmei\}@cse.unsw.edu.au \\ 2 School of Computing Sciences \\ University of Technology, Sydney, NSW 2009, Australia \\ liu@socs.uts.edu.au
}

\begin{abstract}
Spatial data mining recently emerges from a number of real applications, such as real-estate marketing, urban planning, weather forecasting, medical image analysis, road traffic accident analysis, etc. It demands for efficient solutions for many new, expensive, and complicated problems. In this paper, we investigate a proximity matching problem among clusters and features. The investigation involves proximity relationship measurement between clusters and features. We measure proximity in an average fashion to address possible nonuniform data distribution in a cluster. An efficient algorithm, for solving the problem, is proposed and evaluated. The algorithm applies a standard multi-step paradigm in combining with novel lower and upper proximity bounds. The algorithm is implemented in several different modes. Our experiment results do not only give a comparison among them but also illustrate the efficiency of the algorithm.
\end{abstract}

Keywords: Spatial query processing and data mining.

\section{Introduction}

Spatial data mining is to discover and understand non-trivial, implicit, and previously unknown knowledge in large spatial databases. It has a wide range of applications, such as demographic analysis, weather pattern analysis, urban planning, transportation management, etc. While processing of typical spatial queries (such as joins, nearest neighbouring, KNN, and map overlays) has been received a great deal of attention for years [23428], spatial data mining, viewed as advanced spatial queries, demands for efficient solutions for many newly proposed, expensive, complicated, and sometimes ad-hoc spatial queries.

Inspired by a success in advanced spatial query processing techniques [2,3,4], [11,12 28, relational data mining 1,26,30], machine learning [9,10,22, computational geometry [27, and statistics analysis [17/29], many research results and system prototypes in spatial data mining have been recently reported [2 5]6 13],

\footnotetext{
^ The work of this author is partially supported by a small ARC
} 
[15 $\left.18 \begin{array}{lllll}15 & 21 & 24\end{array}\right]$. The existing research does not only tend to provide system solutions but also covers quite a number of special purpose solutions to ad-hoc mining tasks. These include efficiently computing spatial association rules 20, spatial data classification and generalization [13 15 2124], spatial prediction and trend analysis [6], clustering and cluster analysis [57 18 25 32], mining in image and raster databases [8], etc.

Clustering has been proven one of the most useful tools to partition and categorize spatial data into clusters for the purpose of knowledge discovery. A number of efficient algorithms $[5 / 7 / 253132$ have been proposed. Consider that the clustering technique might be too expensive to apply to approaching ad-hoc spatial data mining tasks. In [18|19], special purpose mining algorithms have been developed, as alternatives to clustering, for solving two ad-hoc problems. The first problem is to find the $k$ closest features surrounding a set of points in two dimensional space. Such a set of points may be either a cluster obtained by a clustering algorithm or an existing spatial object (e.g. a residential area) in the database, while a feature is a polygon. The second problem in 18 is to compute the commonality among $n$ sets of points (e.g. $n$ residential areas), provided that their $k$ closest features are pre-computed.

To complement the research in [1819], in this paper we study the following ad-hoc proximity matching problem (PM) among $n$ sets of points:

Suppose that in two dimensional space, $n$ sets of points and $m$ sets of polygons are given. Regarding to a specific set $C$ of points, find the sets of points such that each cluster $C^{\prime}$ meet the proximity matching condition - the "shortest distance" from $C^{\prime}$ to each set $\pi$ of polygons is not greater than that between $C$ and $\pi$. Further, if no sets of points meet the proximity matching condition then the "best" approximate solution is computed.

PM problem has a number of useful real applications. For instance, in realestate spatial data, a set of points represent a residential area where each point represents a land parcel; a polygon corresponds to a vector representation of feature, such as a lake, golf course, school, motor way, etc. In this application, a house buyer or a real-estate developer may want to purchase a property in a well-known area $C$ because of the proximity relationships to certain surround features but may not be able to do it due to either no property available in $C$ or a budget limit. Therefore, the purchaser has to alternatively choose the available and affordable residential areas most similar to $C$ with respect to these proximity relationships. Other applications include road traffic accident investigation, criminal analysis, etc.

PM will be formally defined in the next session. In PM, we assume that the "shortest distance" between a set of points and a set of polygon has not been pre-computed, nor stored in the database. Further, such a shortest distance will be defined in average sense to reflect non-uniform data distribution. These differentiate PM with KNN [2,13], the problem of searching commonalities among $n$ sets of points [18], and incremental distance join problem [16]. 
A naive way to solve PM is to first precisely compute the distance information between each set of points and each set of polygons, and then to solve PM. However, in practice there may be many sets of points far from being part of solution to PM. Motivated by this, our algorithm adopts a standard multi-step technique [2:4 18 20] in combining with novel and powerful pruning conditions to filter out uninvolved features and sets of points. The algorithm has been implemented in several different modes for performance evaluation. Our experiments clearly demonstrates the efficiency of the algorithm.

The rest of the paper is organized as follows. In section 2, we present a precise definition of PM as well as brief an adopted spatial database architecture. Section 3 presents our algorithm for solving PM. Due to the length limitation, in this paper we sketch only the proofs of our theoretical results, and the interested readers may refer to our full paper [23] for the detailed proof. Section 4 reports our experiment results. In section 5 , a discussion is presented regarding various modifications of our algorithm. This is followed by the conclusions and remarks.

\section{Preliminary}

In this section we precisely define the PM problem. A feature $F$ is a simple and closed polygon [27] in the 2-dimensional space. A set $C$ of points in the two dimensional space is called cluster for notation simplicity. Following [18, we assume that in PM a cluster is always outside [27] a feature. Note that this assumption may support many real applications. For instance, in real-estate data, a cluster represents a set of land parcel, and a feature represents a manmade or natural place of interest, such as lake, shopping center, school, park, entertainment center, etc. Such data can be found in many electronic maps in a digital library.

To efficiently access large spatial data (usually tera-bytes), in this paper we adopt an extended-relational and a SAND (spatial-and-non-spatial database) architecture [3]. That is, a spatial database consists of a set of spatial objects and a relational database describing non-spatial properties of these objects. For instance, a set of electronic data describing Sydney metropolitan area may be organized as follows.

- SUBURB (name, \#houses, \#units, average_price, ..., g_des),

- GOLF COURSE (name, \#holes, ..., g_des),

- SCHOOL (name, type, ..., g_des),

- BEACH (name, type, ..., g_des).

In the above database schemata, the attribute $g_{-} d e s$ represents a spatial object, which is either a set of points or a polygon in PM. In order to achieve efficient access, in SAND the attribute $g_{-}$des stores only a pointer in the relational table, pointing to the actual spatial object description. Below shows an example of PM: 
Example 1. select $s .{ }^{*}$

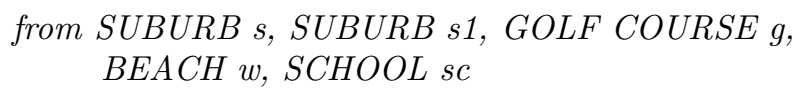

Example 1 is to find the suburbs with area average house price less than $\$ 400,000$, such that their individual shortest distances to the golf courses with 9 holes, to private schools, and to beaches are respectively smaller than those between the suburb Randwick and the features. If such suburbs do not exist then the suburbs most approximately meet the proximity matching conditions will be reported.

Taking the above query as an example, we now formally define PM. In PM, the input consists of:

- a cluster $C_{0}$ (e.g. the suburb Randwick in Example 1),

- a set $S$ of clusters (e.g. the suburbs with average_price not greater than $\$ 400,000$ in Example 1),

- a set $\Pi=\left\{\pi_{j}: 1 \leq j \leq m\right\}$ of groups of features (e.g. in Example 1, $m=3$, $\pi_{1}$ is the set of golf courses with 9 holes, $\pi_{2}$ is the set private schools, and $\pi_{3}$ is the set of beaches).

Given a feature $F$ and a point $p$ outside $F$, the length of the actual (working or driving) shortest path from $p$ to $F$ is too expensive to compute in the presence of tens of thousands of different roads. In PM, we use the shortest Euclidean distance from $p$ to a point in the boundary of $F$, denoted by $d(p, F)$, to reflect the geographic proximity relationship between $p$ and $F$. We believe that on average, the length of an actual shortest path can be reflected by $d(p, F)$. We call $d(p, F)$ the distance between $p$ and $F$. Note that if $F$ degenerates to a point $p^{\prime}$ then $d\left(p, p^{\prime}\right)$ means the Euclidean distance between them; and $F$ may also degenerate to a line. Moreover, for the purpose of computing lower and upper proximity bounds in Section 3, we need to extend the definition of $d(p, F)$ to cover the case when $p$ is inside or on the boundary of $P$; that is, $d(p, F)=0$ if $p$ is inside $P$.

A proximity value between a cluster $C$ and a feature $F$ can be defined in a number of ways. We may define it by the shortest distance between the "boundary" of $C$ and the boundary of $F$. However, as points in $C$ admit an arbitrary distribution, such a proximity value may not be the majority consensus from $C$; this was shown in [18]. We use the following average proximity value to quantitatively model the proximity relationship between $F$ and $C$ :

$$
A P(C, F)=\frac{1}{|C|} \sum_{p \in C} d(p, F)
$$

Consider that in PM a set $\pi$ of features normally means a set of the same kind of features. We define the distance between a set $\pi$ of features and a cluster 
$C$ to be the smallest average proximity between $C$ and a $F \in \pi$, and it is denoted by $D(C, \pi)$. That is,

$$
D(C, \pi)=\min _{F \in \pi}\{A P(C, F)\}
$$

As mentioned earlier, if in PM no cluster meets the above requirements, then the proximity matching needs to find clusters that achieve the requirement most; in this case, we rank the importance of a set $\pi_{j}$ of features by a positive value $w_{j}$. The more important a feature $\pi_{j}$ is, the larger $w_{j}$ is. The $w_{j}$ can be assigned by either a user or the system default. Therefore, a set $\left\{w_{j}: 1 \leq j \leq m\right\}$ of positive values is also part of the input of PM. PM can now be modeled as to find the clusters $C$ in $S$ such that the following goal function is minimized.

$$
P M_{C_{0}}(C, \Pi)=\sum_{j=1}^{m} w_{j} \operatorname{pm}\left(D\left(C, \pi_{j}\right), D\left(C_{0}, \pi_{j}\right)\right)
$$

where,

$$
p m\left(D\left(C, \pi_{j}\right), D\left(C_{0}, \pi_{j}\right)\right)= \begin{cases}0 & \text { if } D\left(C, \pi_{j}\right) \leq D\left(C_{0}, \pi_{j}\right) \\ D\left(C, \pi_{j}\right)-D\left(C_{0}, \pi_{j}\right) & \text { otherwise }\end{cases}
$$

\section{Algorithms for Solving PM}

In this section, we present an efficient algorithm for solving PM. The algorithm is denoted by CPM, which stands for Computing the Proximity Matching.

An immediate way (brute-force) to solve $\mathrm{PM}$ is to 1 ) compute $A P(C, F)$ firstly for each pair of a cluster $C$ and a feature $F, 2)$ secondly compute $D\left(C, \pi_{j}\right)$ for every pair of a $C$ and a $\left.\pi_{j}, 3\right)$ thirdly compute $P M_{C_{0}}(C, \Pi)$ for each cluster $C$ in $S$, and 4) finally find the clusters $C$ with the smallest values of $P M_{C_{0}}(C, \Pi)$. Note that $A P(C, F)$ can be easily computed in $O(|C||F|)$ according to the definition of $A P(C, F)$; and it is the dominant cost. Though the brute-force approach runs in quadratic time regarding the input size, there may be hundreds clusters and tens of thousands features involved in the computation. Moreover, each cluster (feature) may have a number of points (edges). This makes the brute-force approach computationally prohibitive in practice; and our experiment results in Section 4 confirm this.

An alternative way to approach PM is to adopt a multi-step paradigm [24], $[18,20$. That is, we firstly apply a coarse and fast computation. Instead of computing the actual value of $A P(C, F)$ in quadratic time, we may compute a lower bound and an upper bound for $A P(C, F)$ in a constant time $O(1)$. By these bounds, for each cluster $C$ in $S$ we can rule out the features in a $\pi_{j}$, which are definitely not closest to $C$; and thus we do not have to precisely compute the average proximity values between these eliminated features and $C$. Secondly, we can deduce a lower bound and an upper bound for each $P M_{C_{0}}(C, \Pi)$ from the 
bounds of $A P$; and then filter out uninvolved clusters. This is the basic idea of our algorithm. In our algorithm CPM, we have not integrated our algorithm into a particular spatial index, such as $R$-trees, $R^{+}$-trees, etc, due to the following reasons:

- There may be no such a spatial index built.

- The PM problem may involve many features from different tables/electronic thematic maps; and thus, spatial index built for each thematic map may be different. This brings another difficulty to make use of spatial indices.

- A feature or a cluster, which is qualified in PM, may be only a part of a stored spatial object; for instance, user can be interested in only certain part of a residential area. This makes a possible existing index based on the stored spatial objects not applicable.

- The paper 18] indicates the existing spatial indexing techniques do not necessarily support well the computation of aggregate distances; the argument should be also applied to average distance computation.

The algorithm CPM consists of the following 5 steps:

Step 1: Read the relevant clusters into buffer.

Step 2: Read features batch by batch into buffer and determine their groups by validating the selection conditions against the relational tables.

Step 3: For each feature $F$ in a $\pi_{j}$, compute lower and upper bounds of $A P(C, F)$ for a cluster $C$. Then determine whether or not $F$ should be kept for the computation of $D\left(C, \pi_{j}\right)$.

Step 4: For each $\pi_{j}$ and each cluster $C$, compute lower and upper bounds for $D\left(C, \pi_{j}\right)$; and then derive lower and upper bounds for (4). Filter out clusters which will not be part of the solution to PM.

Step 5: Apply the above brute-force method to the remaining clusters and their associated features to solve PM.

In the next several subsections we detail the algorithm step by step. Clearly, a success of the algorithm CPM largely relies on how good the lower and upper bounds of $A P$ are. The goodness of lower and upper bounds means two things: 1) the bounds should be reasonably tight, and 2) the corresponding computation should be fast. We first present the lower and upper bounds.

Note that for presentation simplicity, the algorithms presented in the paper are restricted to the case when features and clusters qualified in PM are stored spatial objects in the database. However, they can be immediately extended to cover the case when a feature or a cluster is a part of a stored object.

\subsection{Lower and Upper Bounds for Average Proximity}

In this subsection, we recall first some useful notation. The barycenter (centroid) of a cluster $C$ is denoted by $b(C)$. A convex 27] polygon encompassing a feature $F$ is called a bounding convex polygon of $F$. The smallest bounding convex polygon of $F$ is called the convex hull [27] of $F$ and is denoted by $P_{F}$. An 
isothetic rectangle is orthogonal to the coordinate axis. The minimum bounding rectangle of $F$ refers to the minimum isothetic bounding rectangle of $F$ and is denoted by $R_{F}$. Similarly, we denote the convex hull of a cluster $C$ by $P_{C}$ and denote the minimum bounding rectangle of $C$ by $R_{C}$. The bounds presented in the subsection are based on either minimum bounding rectangles or convex hulls.

Given a $R_{C}$ and a $R_{F}$, an immediate idea is to use the shortest distance and the longest distance between $R_{C}$ and $R_{F}$ to respectively represent a lower bound and an upper bound of $A P(C, F)$. However, this immediate idea has two problems. The first problem is that when two rectangles intersect with each other (note that in this case $C$ and $F$ do not necessarily have an intersection), the shortest and longest distances between $R_{C}$ and $R_{F}$ are not well defined. The second problem is that the bounds may not be very tight even if the two rectangles do not intersect. These also happen similarly for convex hulls. Below, we present new and tighter bounds.

Our lower bound computation is based on the following Lemma.

Lemma 1. $\sum_{i=1}^{K} \sqrt{x_{i}^{2}+y_{i}^{2}} \geq \sqrt{\left(\sum_{i=1}^{K} x_{i}\right)^{2}+\left(\sum_{i=1}^{K} y_{i}\right)^{2}}$

Proof: It can be immediately verified that the inequality holds when $K=2$. By mathematical induction, we can prove the Lemma.

From Lemma 1, Theorem 2 immediately follows.

Theorem 2. Suppose that $C$ is a cluster, $F$ is a feature, and $P$ is either the convex hull or the minimum bounding rectangle of $F$. Then, $A P(C, F) \geq d(b(C), P)$; in other words $d(b(C), P)$ is a lower bound of $A P(C, F)$.

Figure 1 gives an example, and shows that our lower bound is tighter than the shortest distance between two rectangles.

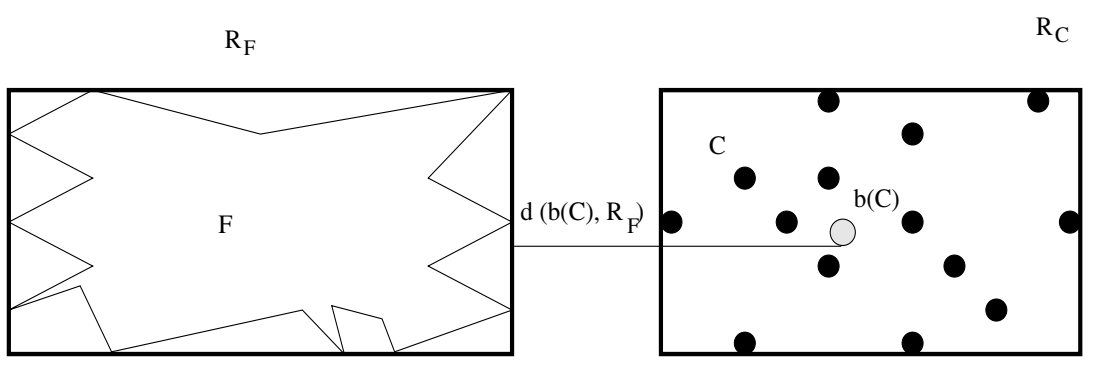

Fig. 1. A lower bound

The $R_{F}$ of a feature $F$ has four edges: the left boundary, right boundary, bottom boundary, and top boundary. Note that each boundary edge $x$ is divided into several line segments (at least two). The two end points of such a line segment are either a) a pair of two adjacent intersection points between $F$ and 
$R_{F}$, or b) a vertex of $R_{F}$ and an intersection point between $F$ and $R_{F}$. We use $H R_{F, x}$ to denote the maximal segment length among these line segments in the boundary edge $x$, where $x \in\{l, r, b, t\}$ respectively represents either the left, or right, or bottom, or top boundary. For each boundary edge $x$, we use $V R_{F, x}$ to denote the maximal length of the perpendicular line segment from a vertex of an edge of $F$, which faces [27] $x$. Figure 2(a) illustrates these concepts.

$$
\mathrm{R}_{\mathrm{F}}
$$

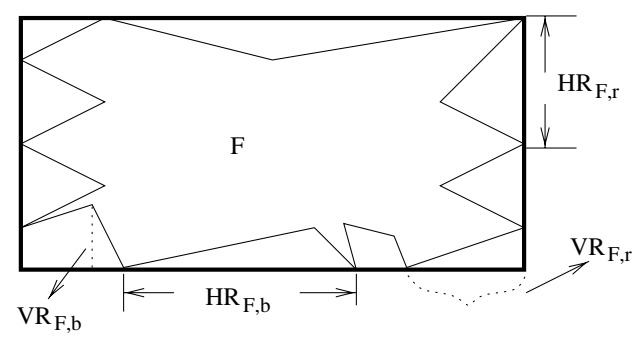

(a)

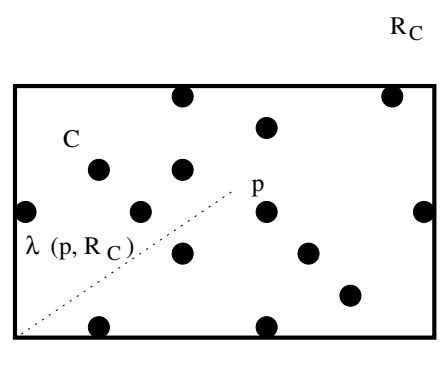

(b)

Fig. 2. Examples

Suppose that $P^{\prime}$ is either the convex hull or the minimum bounding rectangle of a cluster $C$, and $p$ is a point inside $P^{\prime}$. We use $\lambda\left(p, P^{\prime}\right)$ to denote the maximal distance between $p$ and a point contained in $P^{\prime}$. Clearly, $\lambda\left(p, P^{\prime}\right)$ is the maximal distance from $p$ to one of the vertices of $P^{\prime}$. Figure 2(b) illustrates this concept for the minimum bounding rectangle.

Below we present two upper bounds respectively for convex hulls, and minimum bounding rectangles.

Theorem 3. Suppose that $P_{C}$ is the convex hull of a cluster $C, p$ is a point inside $P_{C}$, and $F$ is a feature. Then $A P(C, F) \leq d(p, F)+\lambda\left(p, P_{C}\right)$.

Sketch of the Proof: The theorem can be verified according to the definitions of $A P, d$, and $\lambda$.

It is clear the right hand side in the inequality of Theorem 3 can be used as an upper bound of $A P$. However, the computation of $d(p, F)$ runs in time $O(|F|)$. The lower bound presented below in Theorem 4 is based on the minimum bounding rectangles and can be computed in constant time, though it is not as tight as that in Theorem 3 First we should note that Theorem 3 also holds if we replace $P_{C}$ by $R_{C}$.

Theorem 4. Suppose that $R_{C}$ of $C$ is given, $R_{F}$ of $F$ is given, and $p$ is a point contained in $R_{C}$. Then

$$
d(p, F)+\lambda\left(p, R_{C}\right) \leq \min _{x \in\{l, r, b, u\}}\left\{\min \left\{H R_{F, x}, V R_{F, x}\right\}+d(p, x)\right\}+\lambda\left(p, R_{C}\right) \cdot(5)
$$

Here $x$ a boundary edge of $R_{F}$. 
Proof: From the definition of $H R$ and $V R$, the theorem can be immediately verified.

Theorem 4 together with Theorem 3 imply the right hand side in the inequality of Theorem 4 is another upper bound of $A P$. Further, it should be clear that this upper bound can be computed in constant time, provided that $H R_{F, x}$ and $V R_{F, x}$ are obtained and $\lambda\left(R_{C}, p\right)$ is computed for a given $p$.

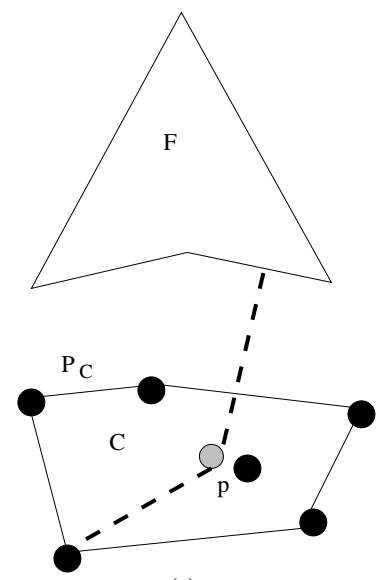

(a)

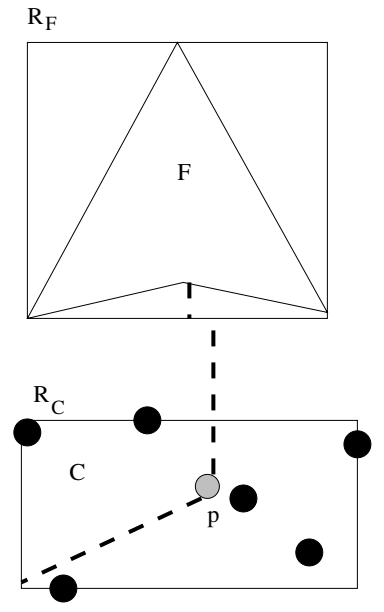

(b)

Fig. 3. Upper bounds

The total lengths of the thick dotted lines in Figure 3(a) and Figure 3(b) respectively show the upper bounds in Theorems 3 and 4 . They also show that our bounds may be tighter than the longest distance between the convex hulls or between the minimum bounding rectangles. However, we cannot generally prove this because the tightness of the bounds depends on the choice of $p$. In our algorithm, we will choose the centroid of a cluster $C$ in the upper bound computation since it has to be used to obtain a lower bound.

In the next several subsections, we present first the algorithm CPM based on the minimum bounding rectangles.

\subsection{Read in Clusters}

In Step 1, we first read in the clusters, specified in the query by a user, into buffer by execution of the data retrieval method 3]; for instance, regarding the query in Example 1 the suburbs with average house price less than $\$ 400,000$ are read into the database by querying the SUBURB table. Then, we compute $R_{C}$, $b(C)$, and $\lambda\left(b(C), R_{C}\right)$ for each cluster $C$. Clearly, this step takes linear time with respect to the total size of clusters. 


\subsection{Read In and Filter Out Features}

This subsection presents Step 2 and Step 3. Consider that the number of the features to be processed may be very large, and each feature may have many edges. It may be impossible to keep all features in buffer all the time. Consequently, features should be read into buffer batch by batch. Once a batch of features are read in, they are first assigned group IDs against users specifications; for in stance, in Example 1 three feature groups are retrieved.

After a feature is assigned a group ID, the algorithm CPM invokes the filtering process in Step 3. It is based on the following lemma.

Lemma 5. Suppose that $C$ is a cluster, and $\pi=\left\{F_{1}, F_{2}, \ldots, F_{k}\right\}$ is a group of features. For $1 \leq j \leq k$, let $L B_{A P}\left(C, F_{j}\right)$ and $U B_{A P}\left(C, F_{j}\right)$ be a lower bound and an upper bound of $A P\left(C, F_{j}\right)$. Then:

$$
\min _{1 \leq j \leq k}\left\{L B_{A P}\left(C, F_{j}\right)\right\} \leq D(C, \pi) \leq \min _{1 \leq j \leq k}\left\{U B_{A P}\left(C, F_{j}\right)\right\} .
$$

Proof: The lemma immediately follows from the definition of $D$.

For notation simplicity, we extend $S$ to include the given $C_{0}$; that is, $S=$ $\left\{C_{i}: 0 \leq i \leq n\right\}$. With respect to each pair of $C_{i}$ and $\pi_{j}$, we use $L B^{i, j}$ to record the minimum value of the lower bounds of $A P\left(C_{i}, F\right)$ for each $F \in \pi_{j}$, and use $U B^{i, j}$ to record the minimum values of the upper bounds. Lemma 5 says that $L B^{i, j}$ and $U B^{i, j}$ are relatively a lower and an upper bound of $D\left(C_{i}, \pi_{j}\right)$.

In our algorithm, we initially set both $L B^{i, j}$ and $U B^{i, j}$ to $\infty$, and then gradually update them when a new feature in $\pi_{j}$ is processed. We use a dynamic array $A_{i, j}$ to store the candidate features in $\pi_{j}$ for computing $A P\left(C_{i}, \pi_{j}\right)$. Each element in $A_{i, j}$ stores the identifier FID of a feature $F$, the obtained lower bound of $A P\left(C_{i}, F\right)$, and the pointer $g_{-}$des that points to the spatial description of the cluster.

Specifically, to process a $F \in \pi_{j}$, CPM firstly computes $R_{F}$ and the values of $V F$ and $H F$ for $R_{F}$; this can done easily by scanning $F$ only once. Secondly, for each $C_{i}$, we check if $F$ should be included in $A_{i, j}$. According to Lemma 5 we add $F$ to $A_{i, j}$ if the obtained lower bound of $A P\left(C_{i}, F\right)$ is less than the current value of $U B^{i, j}$. Further, we should update $L B^{i, j}$ and $U B^{i, j}$ each time after $F$ is added to $A_{i, j}$; and then check $A_{i, j}$ to determine if some features in $A_{i, j}$ should be removed due to an update of $U B^{i, j}$. To prevent unnecessary scan of $A_{i, j}$ each time after $U B^{i, j}$ is updated, we also record the maximum value of the lower bounds of $A P\left(C_{i}, F\right)$ among the features $F$ in the current $A_{i, j}$; it is recorded by $q^{i, j}\left(q^{i, j}\right.$ is initially zero).

For example, suppose that the features $F_{1}, F_{2}, F_{3}$, and $F_{4}$ are in $\pi_{1}$. Their lower and upper bounds of $A P$ values against a cluster $C_{1}$ are $(4,5)$ for $F_{1}$, $(6,7)$ for $F_{2},(3,6)$ for $F_{3}$, and $(3,3.5)$ for $F_{4}$; see Figure 4. Initially, $L B^{1,1}=$ $U B^{1,1}=\infty$, and $q^{1,1}=0$. Suppose that $F_{1}$ is first processed. We add $F_{1}$ to $A_{1,1}$ by recording its ID and the lower bound; and then $L B^{1,1}=4, U B^{1,1}=5$, and $q_{1,1}=4$. Next, $F_{2}$ is processed. $F_{2}$ should not be included in $A_{1,1}$ because $6>U B^{1,1}$. However, when $F_{3}$ is processed thirdly, $F_{3}$ should be added in $A_{1,1}$. 
Consequently, $L B^{1,1}=3, U B^{1,1}=5, q^{1,1}=4$. While processing $F_{4}$, we find that $F_{4}$ should be added to $A_{1,1}$. Accordingly, $L B^{3,3}=3, U B^{1,1}=3.5$, and $q^{1,1}=4$. Then, since $q^{1,1}>U B_{1,1}$ we should check $A_{1,1}$ to delete $F_{1}$ from $A_{1,1}$.

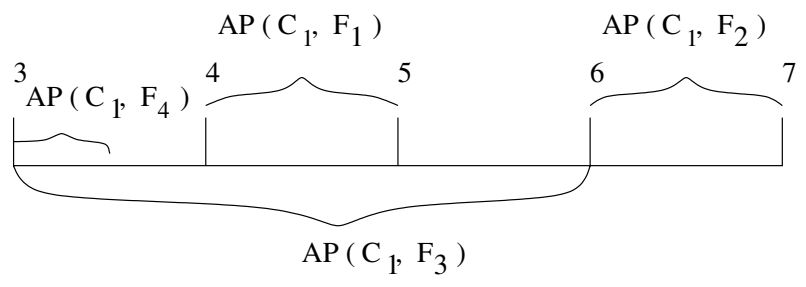

Fig. 4. Filter Out Features

More precisely, to process a feature $F \in \pi_{j}$, the above processes can be presented by the following pseudo codes:

compute $R_{F}, V F_{R, x}$ and $H F_{R, x} ; / * x=\{l, r, b, t\} * /$

for each cluster $C_{i}$ do \{

$L B_{A P}\left(C_{i}, F\right) \rightarrow a_{1}$ (the lower bound); $U B_{A P}\left(C_{i}, F\right) \rightarrow a_{2}$ (the upper bound);

if $a_{1}<U B^{i, j}$ then \{

$F \rightarrow A_{i, j}$

$\min \left\{a_{1}, L B^{i, j}\right\} \rightarrow L B^{i, j}$

$\min \left\{a_{2}, U B^{i, j}\right\} \rightarrow U B^{i, j}$

$\max \left\{q^{i, j}, a_{1}\right\} \rightarrow q^{i, j}$

if $q^{i, j} \geq U B^{i, j}$ then \{

remove features $F$ from $A_{i, j}$ if $L B_{A P}\left(C_{i}, F\right) \geq U B^{i, j}$;

\}

re-compute $q^{i, j}$ from the remaining features in $\left.A_{i, j} ;\right\}$

\}

Once a batch of features are processed by the above procedure, we do not keep them in buffer if no space is left for the next batch of features. In this situation, we will need to read in again the features not filtered out after Step 4, so that we can process Step 4; this is why we want to keep the pointer g_des for each feature object.

\subsection{Filter Out Clusters}

This subsection describes Step 4. After the computation in last subsection, we obtained $L B^{i, j}$ and $U B^{i, j}$ for each pair of $C_{i}$ and $\pi_{j}$. By Lemma 5, we have:

$$
L B^{i, j} \leq D\left(C_{i}, \pi_{j}\right) \leq U B^{i, j}
$$


Note that from the definition, $\operatorname{pm}\left(D\left(C_{i}, \pi_{j}\right), D\left(C_{0}, \pi_{j}\right)\right)=0$ if $D\left(C_{i}, \pi_{j}\right) \leq$ $D\left(C_{0}, \pi_{j}\right)$. This immediately implies that $\operatorname{pm}\left(D\left(C_{i}, \pi_{j}\right), D\left(C_{0}, \pi_{j}\right)\right)=0$ if one of the following two conditions applies:

1. $U B^{i, j} \leq L B^{0, j}$, or

2. $U B^{i, j} \leq D\left(C_{0}, \pi_{j}\right)$

Thus, intuitively $p m\left(D\left(C_{i}, \pi_{j}\right), D\left(C_{0}, \pi_{j}\right)\right)$ is bounded by $U B^{i, j}-L B^{0, j}$. Further, it cannot be smaller than the minimum distance between the two closed intervals: $\left[L B^{i, j}, U B^{i, j}\right]$ and $\left[L B^{0, j}, U B^{0, j}\right]$. The intuition can be immediately verified and is stated in Lemma 6] Let

$$
P^{i, j}= \begin{cases}U B^{i, j}-L B^{0, j} & \text { if } U B^{i, j}>L B^{0, j} \\ 0 & \text { otherwise }\end{cases}
$$

and let

$$
\alpha^{i, j}= \begin{cases}L B^{i, j}-U B^{0, j} & \text { If } L B^{i, j}>U B^{0, j} \\ 0 & \text { otherwise }\end{cases}
$$

Lemma 6. $p m\left(D\left(C, \pi_{j}\right), D\left(c_{i}, \pi_{j}\right)\right) \leq P^{i, j}$, and $p m\left(D\left(C, \pi_{j}\right), D\left(c_{i}, \pi_{j}\right)\right) \geq \alpha^{i, j}$ for each pair of $C_{i}$ and $\pi_{j}$,

Sketch of the Proof: Prove the theorem by applying (6) and the above two conditions.

Lemma 6 gives us a lower and upper bound of $P M_{C_{0}}\left(C_{i}, \Pi\right)$ for each $C_{i}$ :

$$
\sum_{j=1}^{m} w_{j} \alpha^{i, j} \leq P M_{C_{0}}\left(C_{i}, \Pi\right) \leq \sum_{j=1}^{m} w_{j} P^{i, j} .
$$

In Step 4, we firstly compute the lower and upper bounds for each cluster $C_{i}$ $(i \neq 0)$, as given in (17). Secondly, we compute the minimum value $\tau$ of the upper bounds among the clusters $C_{i}$ in $S$ but $i \neq 0$. Thirdly, we scan $S$ to filter out clusters $C_{i}$ if $\sum_{j=1}^{m} w_{j} \alpha^{i, j}>\tau$. This procedure runs in time $O(n m)$.

\subsection{Precise Computation}

This subsection describes Step 5. After pruning the clusters, the information of remaining features for solving PM is kept in each $A_{i, j}$. We need to read in these features again to perform the precise computation. Since two different $A_{i, j}$ s may keep a same feature as a candidate, to efficiently read in required features we use a hashing method. A hash table $H$ is created against feature ID - FID. We scan $A_{i, j}$ one by one to execute the following two steps.

Step 1: For each feature $F \in A_{i, j}$, hash its FID into an $H$ entry and then determine if its spatial description is already in $H$. 
Step 2: If the spatial description of $F$ is not in $H$, then read it into buffer using $g_{-} d e s$ and store it in $H$. Then, compute $A P\left(C_{i}, F\right)$ for each remaining $C_{i}$.

After computing all $A P$ values, we apply the remaining step of the brute-force method to the existing clusters to solve the problem. Note that before starting Step 5, we also check a special case - if $\alpha^{i, j}=P^{i, j}$ for all remaining clusters and features then CPM does not have to process Step 5 but outputs the remaining clusters as the solution.

\subsection{Complexity of CPM}

In this subsection, we analyze the complexity of CPM. Step 1 takes linear time with respect to the total sizes of clusters; that is $O\left(\sum_{i=0}^{n}\left|C_{i}\right|\right)$. Step 2 and Step 3 take time $O\left(n \times \sum_{j=1}^{m}\left|\pi_{j}\right|\right)$. Step 4 takes time $O(n m)$, while step 5 takes time $O\left(\sum_{\forall C_{i}, \forall F \in A_{i, j}}\left|C_{i}\right||F|\right)$ for the remaining clusters $C_{i}$.

Note that the brute-force algorithm runs in time $O\left(\sum_{\forall C, \forall F}|C||F|\right)$. It should be clear that in practical, the time complexity of the brute-force method is much higher than that of CPM. This is confirmed by our experiment results in Section 4 .

\subsection{Different Modes of CPM}

The above mode of CPM uses only the minimum bounding rectangles; and it is denoted by CPM-R.

An alternative mode to CPM-R is to use a multiple-filtering technique [2]13 18]:

- first the minimum bounding rectangles are used in Steps 3 and 4, and then

- the convex hulls for features and clusters are adopted to repeat Steps 3 and 4 before processing Step 5 .

It is denoted by CPM-RH. In CPM-RH, we employ the divide and conquer algorithm [27] to compute convex hulls for clusters. To compute a convex hull for a feature (simple polygon), we employ the last step in Graham's scan [27], which runs in linear time. We use the upper bound of $A P$ in Theorem 3 to implement the procedure in Section 3.3 for convex hulls.

Another alternative mode to CPM-R is to use only the convex hulls instead of the minimum bounding rectangles. We denote this mode by CPM-H.

In next section, we will report our experiment results regarding the performances of the brute-force algorithm, CPM-R, CPM-RH, and CPM-H.

\section{Experiment Results}

The brute-force algorithm and the three different modes of CPM have been implemented by $\mathrm{C}++$ on a Pentium I/200 with $128 \mathrm{MB}$ of main memory, running Window-NT 4.0. In our experiments, we evaluated the algorithms for efficiency 
and scalability. Our performance evaluation is basically focused on Step 3 onwards, because the methods 3 . of reading in clusters and features are not our contribution. Therefore, in our experiment we record only the CPU time but exclude I/O costs.

We developed a program to generate a benchmark. In the program, we first use the following random parameters to generate rectangles, such that a rectangle may intersect with at most another rectangle:

- $M$ gives the number of rectangles, and

- $w_{i d}$ controls the width of a rectangle $R$ and $h_{R}$ controls the height of $R$.

More specifically, we first generate $M$ rectangles $R$ with a random width wid $_{R}$ and a random height $h_{R}$, where $1 \leq w_{i} d_{R}, h_{R} \leq 1000$. The generated rectangles are randomly distributed in the 2 -dimensional space, and intersect with at most another rectangle. To generate $n$ clusters, we randomly divide the whole region into $n$ disjoint sub-regions and choose one rectangle from each region. We use a random parameter $N C$ to control the average number of generated points in each rectangle among the chosen $n$ rectangles. These give $n$ clusters. The remaining $M-n$ rectangles correspond to features. We use another random parameter $N F$ to control the average number of the vertices (points) generated in each remaining rectangle.

Note that if $R$ intersects with $R^{\prime}$, we actually generate the points respectively in $R$ and $R^{\prime}-R$ if $R^{\prime}$ is not included in $R$. Further, in each rectangle $R$ corresponding to a feature, we apply a Graham's scan-like algorithm [27] to produce a simple polygon connecting each vertex in $R$; the generated simple polygon is used as a feature. Therefore, in our benchmark we have two kinds of spatial objects - clusters and features.

In the experiments below, we adopt a common set of parameters: 1) a feature has 150 edges on average, 2) a cluster has 300 points on average, and 3) the features are grouped into 20 groups.

In our first experiment, we generate a database with 10 clusters and 1000 features. The experiment results are depicted in Figure 5 where the algorithm CPM-R, CPM-H, and CPM-RH are respective abbreviated to "R", "H", and "R-H".

From the first experiment, we can conclude that the brute-force is practically very slow. Another appearance is that $\mathrm{H}$ is slower than $\mathrm{R}$ and $\mathrm{R}-\mathrm{H}$ due to the fact that in $\mathrm{H}$, the computation of lower and upper bounds for each pair of cluster and feature does not take constant time. Intuitively, $\mathrm{H}$ should be significantly slower than $\mathrm{R}$ and $\mathrm{R}-\mathrm{H}$ when the number of clusters and features increases; this has been confirmed by the second experiment.

The second experiment has been undertaken through two "dimensions":

- Fix the number of features to be 1000, while the number of clusters varies from 5 to 20. The results are depicted in Figure 6.

- Fix the number of clusters to be 200, while the number of features varies from 1000 to 10000. The results are depicted in Figure[7. 


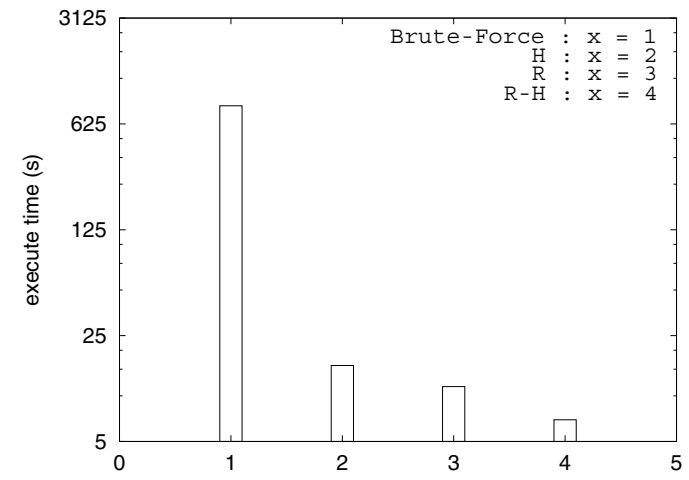

(a) histogram presentation

\begin{tabular}{|c|c|}
\hline Algorithm & $\begin{array}{c}\text { Average Run } \\
\text { Time (s) }\end{array}$ \\
\hline Brute-force & 823.514 \\
\hline H & 15.863 \\
\hline R & 11.516 \\
\hline R-H & 6.95 \\
\hline
\end{tabular}

(b) table presentation

Fig. 5. A Comparison among four algorithms

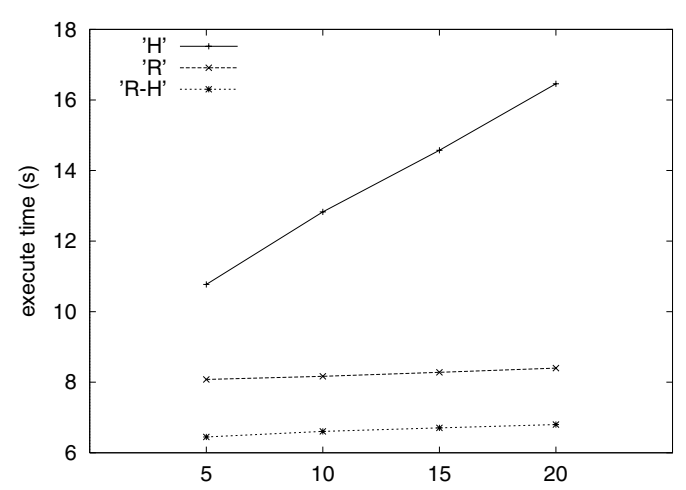

(a) graph presentation

\begin{tabular}{|c|c|l|}
\hline \multirow{3}{*}{ \#CL } & Algorithm & $\begin{array}{c}\text { Average Run } \\
\text { Time (s) }\end{array}$ \\
\hline \multirow{3}{*}{5} & $\mathrm{H}$ & 10.772 \\
\cline { 2 - 3 } & $\mathrm{R}$ & 8.079 \\
\cline { 2 - 3 } & $\mathrm{R}-\mathrm{H}$ & 6.447 \\
\hline \multirow{3}{*}{10} & $\mathrm{H}$ & 12.827 \\
\cline { 2 - 3 } & $\mathrm{R}$ & 8.168 \\
\cline { 2 - 3 } & $\mathrm{R}-\mathrm{H}$ & 6.606 \\
\hline \multirow{3}{*}{15} & $\mathrm{H}$ & 14.575 \\
\cline { 2 - 3 } & $\mathrm{R}$ & 8.28 \\
\cline { 2 - 3 } & $\mathrm{R}-\mathrm{H}$ & 6.706 \\
\hline \multirow{3}{*}{20} & $\mathrm{H}$ & 16.458 \\
\cline { 2 - 3 } & $\mathrm{R}$ & 8.399 \\
\cline { 2 - 3 } & $\mathrm{R}-\mathrm{H}$ & 6.8 \\
\hline
\end{tabular}

(b) table presentation

Fig. 6. A Comparison among H, R, and R-H

Note that in the second experiment, we run $R, H$, and $R-H 40$ times against each database. This experiment, together with the first experiment, also demonstrates that $R-H$ is faster than $H$ on average.

In the third experiment, to test the scalability we vary the database size from 1000 features to 50000 features but fix the the number of clusters to be 200. For each database, we run both $\mathrm{R}$ and $\mathrm{R}-\mathrm{H} 40$ times against each database. Figure 8 illustrates our experiment results.

The three conducted experiments suggest that our algorithm is efficient and scalable. Secondly, we can see that though an application of convex hulls to our filtering procedures is more accurate than an application of minimum rectangles, 
but it is too expensive to use directly. The best use of convex hulls should follow an application of minimum bounding rectangles.

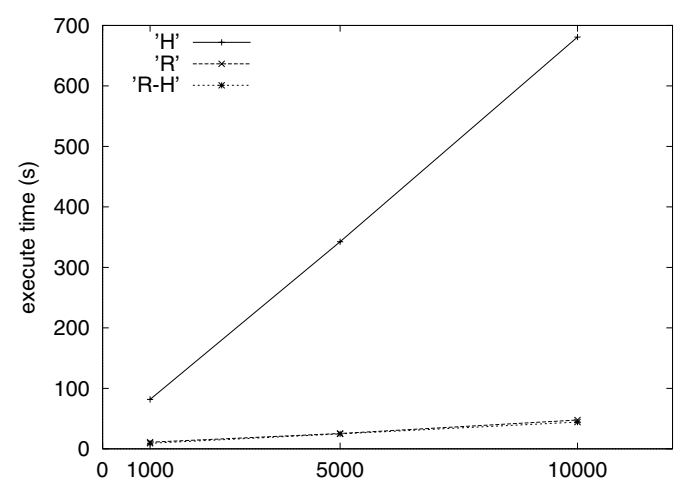

(a) graph presentation

\begin{tabular}{|c|c|c|}
\hline \multirow{4}{*}{ \#FE } & Algorithm & $\begin{array}{c}\text { Average Run } \\
\text { Time (s) }\end{array}$ \\
\hline \multirow{4}{*}{1000} & $\mathrm{H}$ & 81.738 \\
\cline { 2 - 3 } & $\mathrm{R}$ & 10.929 \\
\cline { 2 - 3 } & $\mathrm{R}-\mathrm{H}$ & 9.267 \\
\hline \multirow{3}{*}{5000} & $\mathrm{H}$ & 342.38 \\
\cline { 2 - 3 } & $\mathrm{R}$ & 25.295 \\
\cline { 2 - 3 } & $\mathrm{R}-\mathrm{H}$ & 24.694 \\
\hline \multirow{3}{*}{10000} & $\mathrm{H}$ & 680.751 \\
\cline { 2 - 3 } & $\mathrm{R}$ & 47.578 \\
\cline { 2 - 3 } & $\mathrm{R}-\mathrm{H}$ & 44.246 \\
\hline
\end{tabular}

(b) table presentation

Fig. 7. Another Comparison among H, R, and R-H

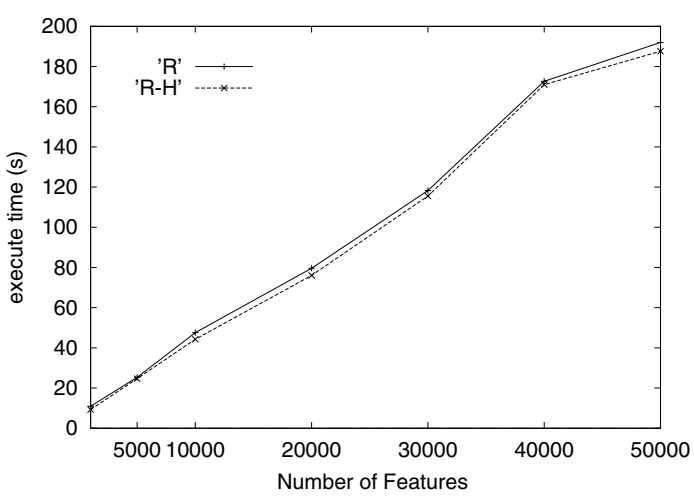

(a) graph presentation

\begin{tabular}{|c|c|c|}
\hline \#FE & Algorithm & $\begin{array}{c}\text { Average Run } \\
\text { Time (s) }\end{array}$ \\
\hline \multirow{2}{*}{1000} & $\mathrm{R}$ & 10.929 \\
\hline & R-H & 9.267 \\
\hline \multirow{2}{*}{5000} & $\mathrm{R}$ & 25.295 \\
\hline & R-H & 24.694 \\
\hline \multirow{2}{*}{10000} & $\mathrm{R}$ & 47.578 \\
\hline & R-H & 44.246 \\
\hline \multirow{2}{*}{20000} & $\mathrm{R}$ & 79.598 \\
\hline & R-H & 76.023 \\
\hline \multirow{2}{*}{30000} & $\mathrm{R}$ & 118.265 \\
\hline & $\mathrm{R}-\mathrm{H}$ & 115.557 \\
\hline \multirow{2}{*}{40000} & $\mathrm{R}$ & 172.628 \\
\hline & R-H & 171.044 \\
\hline \multirow{2}{*}{50000} & $\mathrm{R}$ & 191.978 \\
\hline & R-H & 187.619 \\
\hline
\end{tabular}

(b) table presentation

Fig. 8. A comparison between $\mathrm{R}$ and $\mathrm{R}-\mathrm{H}$ 


\section{Discussion}

The problem PM and the algorithm CPM may be either generalized or constrained according to various applications. In this section, we present a discussion on these issues.

A slight modification of the algorithm CPM can be applied to the case where we specify the proximity matching conditions directly use the shortest distances instead of specifying a given cluster. For instance, in Example 1 we may directly specify that such suburbs are within $5 \mathrm{~km}$ away from a private school, $6 \mathrm{~km}$ away from a beach, and $2 \mathrm{~km}$ away from a golf course, instead of comparing to the suburb Randwick.

Another modification of the problem is to define $\operatorname{pm}\left(D\left(C, \pi_{j}\right), D\left(C_{0}, \pi_{j}\right)\right)$ as $\left|D\left(C, \pi_{j}\right)-D\left(C_{0}, \pi_{j}\right)\right|$. It can be shown [33] that our algorithms can be immediately modified accordingly to resolve this.

Our results and discussions, so far, are limited to the Euclidean distance. Note that the upper bounds presented in Section 3.1 are based on a triangular inequality in the Euclidean distance; that is $d\left(p_{1}, p_{2}\right) \leq d\left(p_{1}, p_{3}\right)+d\left(p_{3}, p_{2}\right)$. Since the triangular inequality is part of the definition of any metric distance, the upper bounds can be applied to any metric space. We should also note that the lower bound presented in Section 3.1 can be obtained in such a metric space that the metric distance $\gamma$ follows the two constraints below:

$-\gamma\left(p_{1}, p_{2}\right)+\gamma\left(p_{3}, p_{4}\right) \geq \gamma\left(p_{1}+p_{3}, p_{2}+p_{4}\right)$, and

$-\gamma\left(c \times p_{1}, c \times p_{2}\right)=|c| \gamma\left(p_{1}, p_{2}\right)$ for any constant $c$.

Consequently, we can extend the problem PM and the algorithm CPM to any metric space where the above two constraints are satisfied. For instance, we can verify that the Manhattan distance [27] satisfied the two constraints; and thus our algorithm can be extended to 2-dimensional Manhattan distance space.

\section{Conclusions}

In this paper, we formalized a new problem (PM) in spatial data mining from real applications. We presented an efficient algorithm based on several novel pruning conditions, as well as various different modes of the algorithm. Our experiment results showed that the algorithm is very efficient and can support a number of real applications where data with huge volume are present.

Further, in Section 5, we showed that our work in this paper can be extended to many other metric spaces.

Note that the PM problem and the algorithm CPM are restricted to the case where a cluster is outside a feature. This restriction may be not generally applicable to some applications; we are now identifying such applications. Besides, we are currently working on the development of indexing techniques to support CPM. Further, a modification of PM to cover the applications where the distance from a cluster to a set of features is not necessarily restricted to one feature in the set seems more complicated; this is our another future study. 


\section{References}

1. R. Agrawal and R. Srikant, Fast Algorithms for Mining Association Rules, Proceedings of the 20th VLDB Conference, 487-499, 1994.

2. M. Ankerst, B. Braunmuller, H.-P. Kriegel, T. Seidl, Improving Adaptable Similarity Query Processing by Using Approximations, Proceedings of the 24th VLDB Conference, 206-217, 1998.

3. W. G. Aref and H. Samet, Optimization Strategies for Spatial Query Processing, Proceedings of the 17th VLDB Conference, 81-90, 1991.

4. T. Brinkhoff, H. P. Kriegel, and R. Schneider, and B. Seeger, Multistep processing of spatial joins, Proc. of ACM SIGMOD, pp. 197-208, 1994.

5. M. Ester, H.-P. Kriegel, J. Sander and X. Xu, A density-based algorithm for discovering clusters in large spatial databases, Proceedings of the Second International Conference on Data Mining KDD-96, 226-231, 1996.

6. M. Este, H.-P. Kriegel, J. Sander, Spatial Data Mining: A Database Approach, SSD'97, LNCS 1262, 47-65, 1997.

7. V. Estivill-Castro and A.T. Murray, Discovering Associations in Spatial Data - An Efficient Medoid Based Approach, Proceedings of the Second Pacific-Asia Conference on Knowledge Discovery, LNAI 394, 110-121, 1998.

8. U. M. Fayyad, S. G. Djorgovski, and N. Weir, Automating the analysis and cataloging of sky surveys, Advances in Knowledge Discovery and Data Mining, AAAI/MIT Press, 1996.

9. U. Fayyad, G. Piatetsky-Shapiro, P. Smyth, and R. Uthurusamy, Eds. Advances in Knowledge Discovery and Data Mining, AAAI/MIT Press, Menlo Park, CA, 1996.

10. D. Fisher, Improving Inference through Conceptual Clustering, Proceedings of 1987 AAAI Conferences, 461-465, 1987.

11. R. H. Guting, An Introduction to Spatial Database Systems, VLDB Journal, 3(4), 357-400, 1994.

12. R. Guttman, A Dynamic Index Structure for Spatial Searching, ACM-SIGMOD International Conference on Management of Data, 47-57, 1984.

13. J. Han, Spatial Data Mining and Spatial Data Warehousing, Invited Talk at SSD'97, 1997.

14. J. Han, Y. Cai, and N. Cercone, Dynamic Generation and Refinement of Concept Hierarchies for Knowledge Discovery in Databases, IEEE Trans. knowledge and Data Engineering, 5, 29-40, 1993.

15. J. Han, K. Koperski, and N. Stefanovic, GeoMiner: A System Prototype for Spatial Data Mining, Proceedings of 1997 ACM-SIGMOD International Conference on Management, 553-556, 1997.

16. G. R. Hjaltason and H. Samet, Incremental Distance Join Algorithms for Spatial Databases, 237-248, Proceedings of the 1998 ACM SIGMOD International Conference on Management of Data, 1998.

17. L. Kaufman and P. J. Rousseeuw, Finding Groups in Data: an Introduction to Cluster Analysis, John Wiley \& Sons, 1990.

18. E. M. Knorr and R. T. Ng, Finding Aggregate Proximity Relationships and Commonalities in Spatial Data Mining, IEEE Transactions on Knowledge and Data Engineering, 8(6), 884-897, 1996.

19. E. M. Knorr, R. T. Ng, and D. L. Shilvock, Finding Boundary Shape Matching Relationships in Spatial Data, SSD'97, LNCS 1262, 29-46, 1997.

20. K. Koperski and J. Han, Discovery of Spatial Association Rules in Geographic Information Databases, Advances in Spatial Databases, Proceeding of 4th Symposium (SSD'95), 47-66, 1995. 
21. K. Koperski, J. Han, and J. Adhikary, Mining Knowledge in Geographic Data, to appear in Communications of ACM.

22. R. S. Michalski, J. M. Carbonnel, and T. M. Mitchell, editors, Machine Learning: An Artificial Intelligence Approach, Morgan Kaufman, 1983.

23. X. Lin, X. Zhou, and C. Liu, Efficient Computation of a Proximity Matching in Spatial Databases, Tec. Report, University of New South Wales, 1998.

24. W. Lu, J. Han, and B. C. Ooi, Knowledge Discovery in Large Spatial Databases, Proceedings of Far East Workshop on Geographic Information Systems, 275-289, 1993.

25. N. Ng and J. Han, Efficient and Effective Clustering Method for Spatial Data Mining, Proceeding of 1994 VLDB, 144-155, 1994.

26. J. S. Park, M.-S. Chen, and P. S. Yu, An Effective Hash-Based Algorithm for Mining Association Rules, Proceedings of 1995 ACM SIGMOD, 175-186, 1995.

27. F. Preparata and M. Shamos, Computational Geometry: An Introduction, SpringerVerlag, New York, 1985.

28. H. Samet, The Design and Analysis of Spatial Data Structures, Addison-Wesley, 1990.

29. G. Shaw and D. Wheeler, Statistical Techniques in Geographical Analysis, London, David Fulton, 1994.

30. H. Toivonen, Sampling Large Databases for Association Rules, Proceedings of 22nd VLDB Conference, 1996.

31. X. Xu, M. Ester, H.-P. Kriegel, Jorg Sander, A Distribution-Based Clustering Algorithm for Mining in Large Spatial Databases, ICDE'98, 324-331, 1998.

32. T. Zhang, R. Ramakrishnan and M. Livny, BIRCH: an efficient data clustering method for very large databases, Proceeding of 1996 ACM-SIGMOD International Conference of Management of Data, 103-114, 1996.

33. X. Zhou, Efficiently Computing Proximity Relationships in Spatial Databases, Master Thesis, University of New South Wales, under preparation, 1999. 